\title{
Radiation Emission and Its Influence on the Motion of Multi-GeV Electrons and Positrons in Strong Crystalline Fields
}

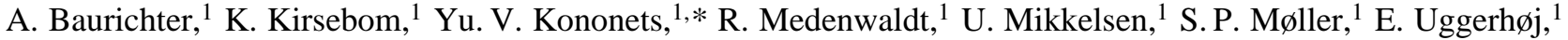 \\ T. Worm, ${ }^{1}$ K. Elsener, ${ }^{2}$ S. Ballestrero, ${ }^{3}$ P. Sona,${ }^{3}$ J. Romano, ${ }^{4}$ S. H. Connell, ${ }^{5}$ J. P. F. Sellschop, ${ }^{5}$ R. O. Avakian, ${ }^{6}$ \\ A. E. Avetisian, ${ }^{6}$ and S. P. Taroian ${ }^{6}$ \\ ${ }^{1}$ ISA, University of Aarhus, DK-8000 Aarhus C, Denmark \\ ${ }^{2}$ CERN, CH-1211 Geneva 23, Switzerland \\ ${ }^{3}$ Dipartimento di Fisica, University of Florence and INFN Sezione di Firenze, I-50125 Florence, Italy \\ ${ }^{4}$ Dipartimento di Fisica, University La Sapienza-Rome and INFN Sezione di Roma, I-00185 Rome, Italy \\ ${ }^{5}$ Schonland Research Centre for Nuclear Sciences, University of Witwatersrand, P.O. Wits, 2050 Johannesburg, South Africa \\ ${ }^{6}$ Yerevan Physics Institute, 375036 Yerevan, Armenia
}

(Received 19 February 1997)

\begin{abstract}
Presented is the first experimental demonstration of a dramatic radiative redistribution in transverse states for multi-GeV electrons/positrons, interacting with strong crystalline fields. Detailed analysis of energy loss, photon multiplicities, and scattering distributions leads to new physical insight into open questions such as radiative cooling/heating of the beams in aligned crystals, validity of the constantfield approximation, radiative capture of random particles ("feed in") into channeled states, and the Landau-Pomeranchuk effect in multiphoton radiation spectra. [S0031-9007(97)04297-X]
\end{abstract}

PACS numbers: 41.75.Ht, 41.60.-m, 78.70.-g

When multi-GeV electrons/positrons traverse single crystals close to axial/planar directions, QED processes are strongly influenced by coherent scattering on many target atoms. Channeling is one type of such coherent processes [1,2]. Along crystal directions the incident ultrarelativistic particles "see" very strong electromagnetic fields $\left(\sim 10^{11} \mathrm{~V} / \mathrm{cm}\right)$. These fields in the particle rest frame must be multiplied by the Lorentz factor $\gamma$ $\left(10^{5}-10^{6}\right)$. Under such conditions radiation emission processes resemble the well-known synchrotron radiation in very strong fields [3]. Further on, the transverse motion of such high-energy projectiles during photon emission is small because the longitudinal radiation formation length, $\ell_{f}$, is assumed to be short, so the crystalline fields are nearly constant during the processes-leading to the so-called constant-field approximation (CFA) $[2,3]$.

The influence of strong fields on QED in the CFA is dependent on the invariant parameter $\chi=$ $\gamma \epsilon / \epsilon_{0}$, where $\epsilon$ is the local field strength, $\epsilon_{0}=$ $m c^{2} / e \lambda_{c}=1.3 \times 10^{16} \mathrm{~V} / \mathrm{cm}$. We use standard notation for electron charge, mass, Compton wavelength, and speed of light. For $\chi \ll 1$ classical electrodynamics can be used, whereas for $\chi \geqslant 1$ a full quantum description is needed - already at $\chi \sim 0.1$ quantum effects begin to appear. In the present experiments $\chi \lesssim 1$.

Soon after the onset of experimental investigations of radiation emission from strong crystalline fields the idea of radiative cooling ( $\mathrm{RC}$ ) [4,5] was brought up in order to describe the experimental data. RC describes the reduction of the particle transverse energy $E_{\perp}$ [see Eq. (1) below] due to photon emission and counteracts the multiple scattering. $\mathrm{RC}$ has also been proposed as a technique to reduce the exit-angle spread [6], i.e., angular cooling in contrast to angular heating (increase of angular spread).
From our experiments [7] we concluded at that time that the photon spectra are very sensitive to multiple scattering and radiative cooling - but only for incident angles smaller than the Lindhard angle $\psi_{c}$.

Later comparisons between our CERN results [8] and new calculations (based on CFA) by Kononets [9] showed large discrepancies between experimental data and calculations, especially for thin targets. It was proposed to change total cross sections for radiation emission and electron scattering.

This led to an upgrade of the CERN detector. The present results from this new setup present a breakthrough in the understanding of the basic QED processes in crystals, i.e., influence of $\mathrm{RC}$ on photon spectra from electrons and positrons, applicability of the CFA, and the influence of effects like the Landau-Pomeranchuk (LP) effect [10]. In this effect the photon yields are suppressed because the multiple scattering angle along the coherence length is larger than the photon emission angle $\gamma^{-1}$ and the coherence is destroyed.

The CERN experiment (NA-43) was performed at the Super Proton Synchrotron using $150 \mathrm{GeV} e^{+} / e^{-}$. The experimental arrangement can be found in Ref. [11], p. 80. In short, three drift chambers give incident and exit angles with respect to the target. A tagging system and lead-glass detectors measure the emitted radiation energy below 140 and above $4 \mathrm{GeV}$, respectively. From photon conversion in a thin $(1 \mathrm{~mm}) \mathrm{Pb}$ converter followed by solid-state detectors the average photon multiplicities are obtained. Two $\langle 110\rangle$ Si crystals (0.6 and $1.4 \mathrm{~mm}$ thick) and a $1.5 \mathrm{~mm}$ thick $\langle 100\rangle$ diamond were used as targets.

In the following some typical experimental results are presented from which the new physics is extracted. Radiation spectra are given as enhancements, 
$\eta\left(E_{r}\right)=\left(L_{\mathrm{rad}} / L\right)\left(E_{r} / N\right)\left(d N / d E_{r}\right)$, i.e., in units of the average Bethe-Heitler yields from amorphous targets. Here $E_{r}$ is the radiated energy, $L$ the target thickness, $N$ the number of projectiles; the radiation length $L_{\mathrm{rad}}=9.4$ and $12.2 \mathrm{~cm}$ for $\mathrm{Si}$ and diamond, respectively. Incident and exit angles, $\theta_{\text {in }}$ and $\theta_{\text {out }}$, are polar angles with respect to the actual crystal axis. Hereby the strong influence of doughnut scattering [2] is eliminated.

In Fig. 1 are shown typical radiation spectra for $150 \mathrm{GeV} e^{+} / e^{-}$together with average photon multiplicities and radiative energy loss. The general picture for radiation emission from multi-GeV $e^{+} / e^{-}$in aligned crystals can be learned from this figure, i.e., in a crystal $\sim 1 \mathrm{~mm}$ thick electrons radiate up to 50 times more energy than in an amorphous target. The best channeled positrons radiate half as much [Fig. 1(d)]. The average photon multiplicities are 5-10 [Fig. 1(c)] leading to mean free paths $\lambda_{f}$ of $0.1-0.2 \mathrm{~mm}$ as compared to $30-50 \mathrm{~mm}$ in amorphous diamond and $\mathrm{Si}$, so $\lambda_{f}$ for emission of $\mathrm{GeV}$ photons is reduced by around 2 orders of magnitude.

Applicability of the CFA. - It can be shown in the CFA that for $\chi \lesssim 1$ the characteristic radiation formation length $\bar{\ell}_{f}$ coincides with the coherence length of char-
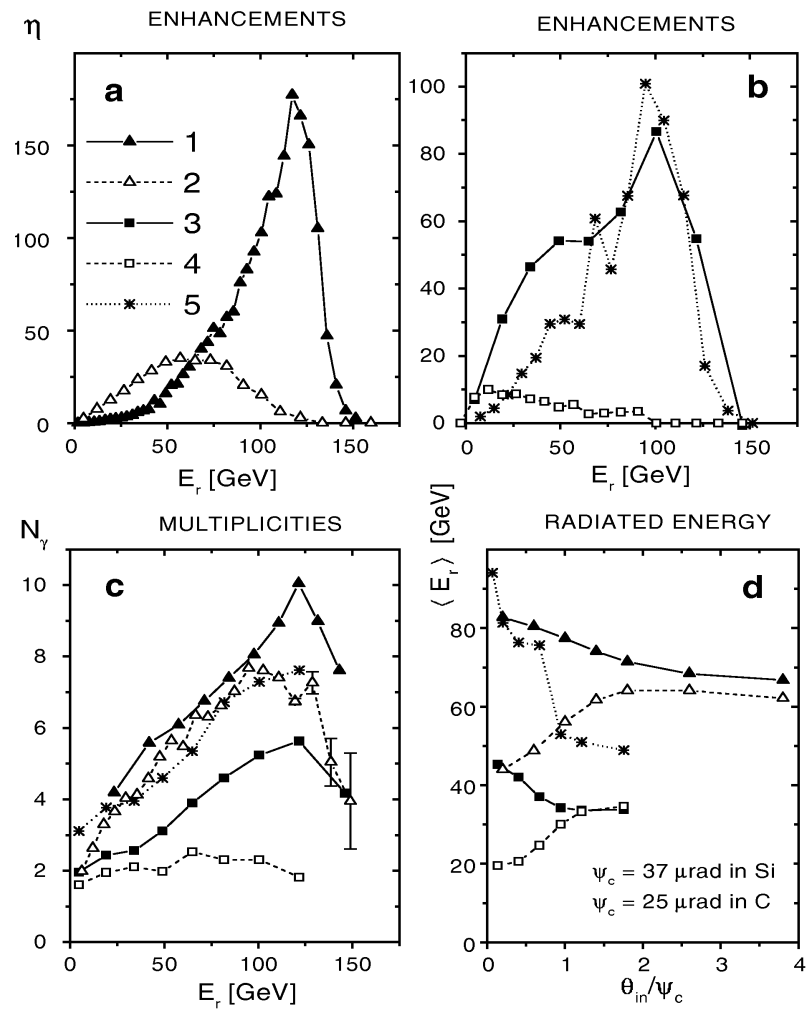

FIG. 1. Radiation spectra (a), (b), photon multiplicities (c), and radiative energy loss (d) for $150 \mathrm{GeV}$ electrons (curves 1 , 3 , and 5) and positrons (curves 2 and 4) incident on the $1.5 \mathrm{~mm}$ thick $\langle 100\rangle$ diamond (curves 1 and 2) and on the $0.6 \mathrm{~mm}$ (curves 3 and 4) and $1.4 \mathrm{~mm}$ (curves 5) thick $\langle 110\rangle \mathrm{Si}$ crystals. The incident-angle regions are $0<\theta_{\text {in }}<10 \mu \mathrm{rad}$ for (a), (b), and $0<\theta_{\text {in }}<20 \mu \mathrm{rad}$ for (c). acteristic photons $\ell_{c}(\hbar \bar{\omega})=2 \lambda_{c} \gamma(E-\hbar \bar{\omega}) / \hbar \bar{\omega}$, where $\hbar \bar{\omega}$ is the average energy of emitted photons. This gives a possibility directly from experimental data to solve the question of the CFA applicability, which has been under debate for many years [12].

From the energy loss and photon multiplicity spectra in Fig. 1 it is easy to estimate a characteristic photon energy $\hbar \bar{\omega}$ in a multiphoton chain emitted by a particle incident close to axial directions. The typical energy loss for electrons in the $0.6 \mathrm{~mm} \mathrm{Si}$ crystal is $\approx 40 \mathrm{GeV}$ and photon multiplicities $\approx 4$ giving $\hbar \bar{\omega} \approx 10 \mathrm{GeV}$. The same value for $\hbar \bar{\omega}$ is found for positrons. The coherence length $\ell_{c}(10 \mathrm{GeV})=3.2 \mu \mathrm{m}$ and has to be compared to a typical longitudinal interaction length $\ell_{\|}$for a particle incident at the Lindhard angle $\psi_{c}$ to the axis. For such particles $\ell_{\|} \approx 1 \AA / \psi_{c} \approx 2.7 \mu \mathrm{m}<\ell_{c}(\hbar \bar{\omega})$. So, during the formation time for a characteristic photon the radiating electrons interact with a continuum crystalline field [1] that changes not only its value but even the sign, leading to violation of the CFA $[2,3]$ in the main region of photon frequencies. This means that the CFA is not a good approximation for the present cases.

It should be pointed out that the situation becomes more dramatic for characteristic photons in thicker Si and diamond crystals. For the present Si targets the experimental results are in good agreement with calculations by Kononets [9], where the simplest phenomenological corrections for radiation probabilities were introduced.

Angular cooling/heating. - In Fig. 2 is seen the first real experimental demonstration of radiative angular cooling/heating for $150 \mathrm{GeV} e^{+} / e^{-}$incident on Si. The average exit angles $\left\langle\theta_{\text {out }}\right\rangle$ are given for increasing energy $E_{e}$ of the exiting particles, which is equivalent to decreasing radiative energy loss $E_{r}$. The full-drawn curves correspond to the Lindhard angles $\psi_{c}\left(E_{e}\right)$ [1]. Four incident-angle regions (two below and two above $\psi_{c}$ ) are presented in the figure. In general, it is found that positrons always come out of the crystals with angles $\left\langle\theta_{\text {out }}\right\rangle>\left\langle\theta_{\text {in }}\right\rangle$. For electrons a similar picture is found for $\left\langle\theta_{\text {in }}\right\rangle<\psi_{c}(E)$, giving $\left\langle\theta_{\text {out }}\right\rangle \approx \psi_{c}\left(E_{e}\right)>\left\langle\theta_{\text {in }}\right\rangle$, whereas for $\left\langle\theta_{\text {in }}\right\rangle>\psi_{c}(E)$ the exit angles are reduced: $\left\langle\theta_{\text {out }}\right\rangle\left\langle\left\langle\theta_{\text {in }}\right\rangle\right.$ for $E_{e} \gtrsim 40 \mathrm{GeV}$, which corresponds to angular cooling. In Si this effect is much stronger than in diamond. Note that multiple scattering alone would increase $\left\langle\theta_{\text {out }}\right\rangle$ by $(1-10) \%$ compared to $\left\langle\theta_{\text {in }}\right\rangle$, depending on $L, E_{e}$, and $\left\langle\theta_{\text {in }}\right\rangle$.

The influence of $\gamma$ emission on the particle motion in a crystal can be seen from the simple classical channeling picture [1,2]. If we assume the $\gamma$ emission to be a localized process in transverse coordinates $\boldsymbol{r}_{\perp}$, then in the continuum approximation the transverse particle energy

$$
E_{\perp}=m \gamma v^{2} \theta^{2} / 2+U\left(\boldsymbol{r}_{\perp}\right)
$$

is conserved between $\gamma$-emission events and the particle velocity $\boldsymbol{v}$ remains unchanged during an emission process. Here $\theta$ is the angle between $\boldsymbol{v}$ and the axis, $U\left(\boldsymbol{r}_{\perp}\right)$ is 

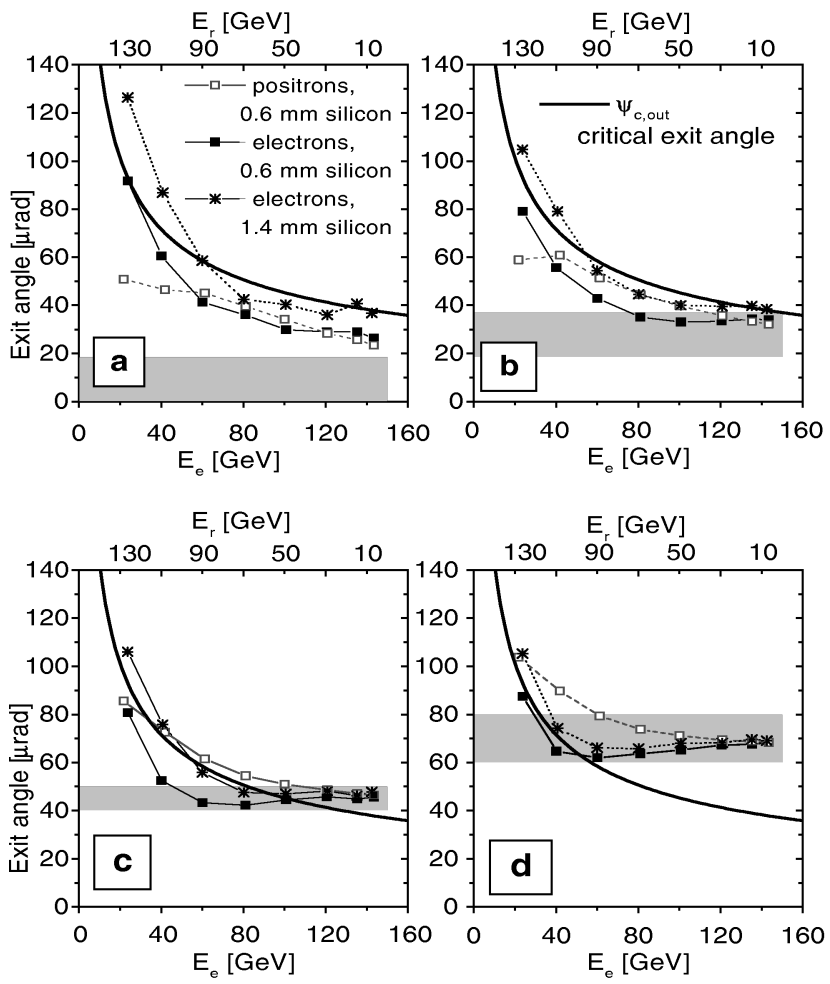

FIG. 2. Average exit polar angles $\left\langle\theta_{\text {out }}\right\rangle$ for $150 \mathrm{GeV}$ electrons and positrons traversing the 0.6 and $1.4 \mathrm{~mm}$ thick $\langle 110\rangle \mathrm{Si}$ crystals, as functions of the exit energy $E_{e}$ for various incidentangle regions (hatched): (a) $0<\theta_{\text {in }}<18.5 \mu \mathrm{rad}$, (b) $18.5<$ $\theta_{\text {in }}<37 \mu \mathrm{rad}$, (c) $40<\theta_{\text {in }}<50 \mu \mathrm{rad}$, and (d) $60<\theta_{\text {in }}<$ $80 \mu \mathrm{rad}$. The full-drawn curves represent the Lindhard angle $\psi_{c}\left(E_{e}\right)$. The minima on the electron curves in (c) and (d) show that RC is dominating over multiple scattering for $E_{e} \gtrsim$ $60 \mathrm{GeV}$.

the continuum potential energy [1]. From this a simple relation between angles before $\left(\theta_{i}\right)$ and after $\left(\theta_{f}\right)$ a $\gamma$ emission is obtained, i.e.,

$$
\begin{aligned}
\left\langle\theta^{2}\right\rangle_{f}= & \left\langle\theta^{2}\right\rangle_{i}+\frac{2}{E-\hbar \omega}\left[U\left(\boldsymbol{r}_{\perp}^{0}\right)-\langle U\rangle_{f}\right] \\
& -\frac{2}{E}\left[U\left(\boldsymbol{r}_{\perp}^{0}\right)-\langle U\rangle_{i}\right],
\end{aligned}
$$

where $\boldsymbol{r}_{\perp}^{0}$ is transverse coordinate of the $\gamma$-emission event, symbols $\langle\ldots\rangle_{i}$ and $\langle\ldots\rangle_{f}$ denote the averaging over initial and final states of the radiating particle, respectively.

For positrons $U\left(\boldsymbol{r}_{\perp}^{0}\right)>0$ and

$$
\left|\langle U\rangle_{i}\right|,\left|\langle U\rangle_{f}\right| \ll\left|U\left(\boldsymbol{r}_{\perp}^{0}\right)\right|
$$

in the main regions of $\boldsymbol{r}_{\perp}^{0}$, which gives

$$
\left\langle\theta^{2}\right\rangle_{f}-\left\langle\theta^{2}\right\rangle_{i} \cong 2\left(\frac{1}{E-\hbar \omega}-\frac{1}{E}\right) U\left(\boldsymbol{r}_{\perp}^{0}\right) .
$$

As $\theta$ is unchanged when the particle crosses a target surface, it follows from Eq. (4) that $\left\langle\theta_{\text {out }}^{2}\right\rangle>\left\langle\theta_{\text {in }}^{2}\right\rangle$, i.e., positrons experience angular heating, even though the transverse energy is reduced.

For electrons $U\left(\boldsymbol{r}_{\perp}^{0}\right)<0$ and here two important incident-angle regions have to be distinguished:

$$
\begin{aligned}
& \text { (a) } \theta_{\text {in }} \ll \psi_{c} \text {; } \\
& \text { (b) } \theta_{\text {in }}>\psi_{c} .
\end{aligned}
$$

A detailed analysis shows that in case (a) the radiation emission and multiple scattering result in capture to highlying states in the potential well, for which $\left\langle\theta_{\text {out }}\right\rangle>\left\langle\theta_{\text {in }}\right\rangle$, i.e., angular heating. In case (b) Eqs. (3) and (4) lead to $\left\langle\theta_{\text {out }}^{2}\right\rangle<\left\langle\theta_{\text {in }}^{2}\right\rangle$, i.e., angular cooling.

By introducing the probability $W_{\text {rad }}\left(\hbar \omega, E, r_{\perp}^{0}\right)$ for emitting a photon of energy $\hbar \omega$ at a point $\boldsymbol{r}_{\perp}^{0}$ and integrating over the 2D unit cell of $\boldsymbol{r}_{\perp}^{0}$ and over all emitted photon energies one gets from Eq. (4) the variation of $\left\langle\theta^{2}\right\rangle$ per unit path $L$ due to radiation emission

$$
\left(\frac{\delta\left\langle\theta^{2}\right\rangle}{\delta L}\right)_{\mathrm{rad}} \approx \frac{2}{\pi} \frac{r_{e}}{S_{\perp}^{0}}\left(\frac{U_{0}}{m c^{2}}\right)^{3} .
$$

Here $r_{e}=e^{2} / m c^{2}, U_{0}$ the potential-energy value at the atomic-row axis, $S_{\perp}^{0}$ the transverse area per atomic row.

Equation (5) is obtained from classical calculations, which is valid for the strong-field parameter $\chi<1$ corresponding to projectile energies $\lesssim 100 \mathrm{GeV}$ in $\mathrm{Si}$ and diamond crystals. The angular cooling/heating [Eq. (5)] has to be compared to the multiple scattering in amorphous targets

$$
\left(\frac{\delta\left\langle\theta^{2}\right\rangle}{\delta L}\right)_{\mathrm{ms}} \cong\left(\frac{21}{E}\right)^{2} \frac{1}{L_{\mathrm{rad}}},
$$

where $E$ is the particle energy in $\mathrm{MeV}$. The ratio of the two quantities Eqs. (5) and (6) is

$$
R \equiv\left|\left(\frac{\delta\left\langle\theta^{2}\right\rangle}{\delta L}\right)_{\mathrm{rad}}\right| /\left(\frac{\delta\left\langle\theta^{2}\right\rangle}{\delta L}\right)_{\mathrm{ms}} \propto \bar{\eta} \psi_{c}^{2},
$$

where $\bar{\eta}$ is the average enhancement of radiation emission. One gets easily $R>1$ for electron energies $\gtrsim 60 \mathrm{GeV}$ in $\langle 110\rangle \mathrm{Si}$, so a strong angular cooling should be observed in good agreement with the results shown in Fig. 2.

The $\psi_{c}^{2}$ dependence in the right-hand side of Eq. (7) explains why the angular cooling effects in the $\langle 100\rangle$ diamond target are much weaker than in $\langle 110\rangle \mathrm{Si}$ in spite of higher values of the enhancement and photon multiplicities (Fig. 1). From Eqs. (5)-(7) it is found that the ratio $R$ along the $\langle 110\rangle$ in diamond must be $\sim 3$ times higher than for the same direction in Si. This effect will be investigated in a coming experiment.

From Figs. 2(c) and 2(d) it is seen that some electrons with $\theta_{\text {in }}>\psi_{c}(E)$ come out with angles $\theta_{\text {out }}<\psi_{c}\left(E_{e}\right)$ and have radiated $\gtrsim 100 \mathrm{GeV}$. Therefore such electrons most probably have been channeled during part of their passage. Thus, radiation emission along $\langle 110\rangle$ in Si leads to a feed-in process for electrons.

The influence of the LP effect can be detected in multiphoton spectra by measuring angular distributions of particles which radiate significantly less than average. These particles, if subject to suppression of the LP type, would undergo anomalously large scattering. 


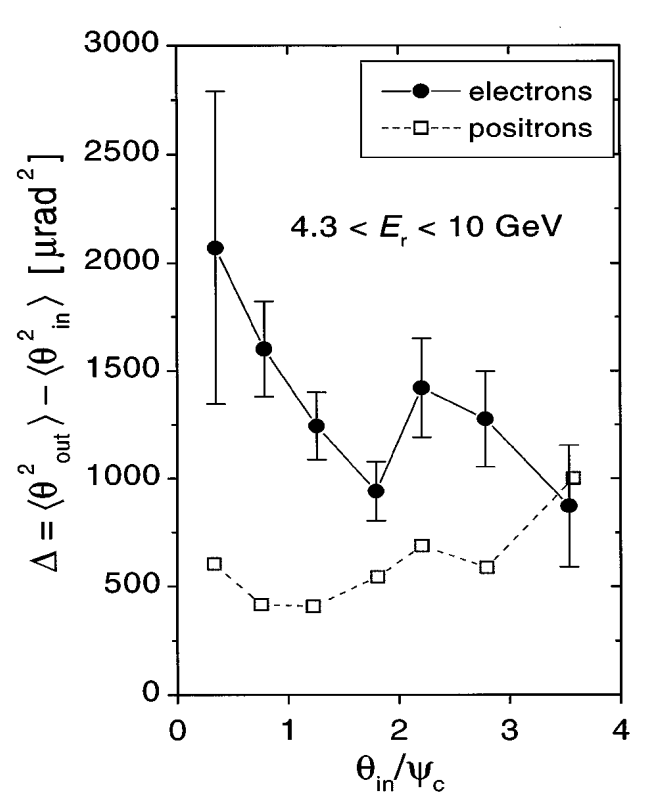

FIG. 3. Difference between averaged exit angles $\left\langle\theta_{\text {out }}^{2}\right\rangle$ and averaged incident angles $\left\langle\theta_{\text {in }}^{2}\right\rangle$ as a function of average incident angle for $150 \mathrm{GeV} e^{-}$and $e^{+}$traversing the $1.5 \mathrm{~mm}$ thick $\langle 100\rangle$ diamond crystal with small radiative losses $E_{r}$.

Such a situation really takes place in the $\langle 100\rangle$ diamond target, as it is seen from Fig. 3, where experimental results for $\Delta=\left\langle\theta_{\text {out }}^{2}\right\rangle-\left\langle\theta_{\text {in }}^{2}\right\rangle$ are presented as functions of $\left\langle\theta_{\text {in }}\right\rangle$ for electrons $\left(\Delta^{-}\right)$and positrons $\left(\Delta^{+}\right)$with radiated energy $4.3<E_{r}<10 \mathrm{GeV}$. Though $\Delta^{+}$is $\sim 3$ times smaller than $\Delta^{-}$at $\theta_{\text {in }} \leq \psi_{c}$, both quantities exceed considerably $\Delta_{\mathrm{ms}}^{\mathrm{am}} \approx 240 \mu \mathrm{rad}^{2}$, characteristic for multiple scattering in an amorphous target of the same thickness $L=1.5 \mathrm{~mm}$. With increasing $\theta_{\text {in }}$ the curves $\Delta^{+}$and $\Delta^{-}$ approach one another, and a characteristic value of $\Delta^{-}$is $\approx 5 \Delta_{\mathrm{ms}}^{\mathrm{am}}$ for $1 \leq \theta_{\mathrm{in}} / \psi_{c} \leq 3$. If we assume suppressed photon yields for $\hbar \omega \leqslant 3 \mathrm{GeV}$, then $\ell_{c}(\hbar \omega)>11 \mu \mathrm{m}$, and it is easy to get from the data presented that for such distances $\Delta^{-}\left(\ell_{c}\right) \cong \Delta^{-}(L) \ell_{c} / L \gtrsim \gamma^{-2}$. These considerations clearly show that the LP effect acts as a mechanism for selecting large-angle scattering events in angular distributions of particles with small radiative losses.

This new possibility for detecting the LP effect is of particular interest for single crystals of heavy elements. Results obtained for radiation from $150 \mathrm{GeV}$ electrons in $0.2 \mathrm{~mm}\langle 111\rangle \mathrm{W}$ are currently being analyzed and will be published later.

In conclusion, the CFA which has been used in most calculations for multi-GeV particles in crystals has its limitations. New theoretical approaches are needed. The strong RC forms a mechanism for capturing particles from above-barrier states into the channeling regime, the efficiency of which grows sharply with increasing energy, and changes radically all the physics of channeling of ultrahigh-energy electrons and positrons. For electrons with incident angles $\theta_{\text {in }}>\psi_{c}$ the RC leads to angular cooling (collimation). It strengthens considerably an effective electron interaction with crystal atomic chains and may open new ways for studying deep inelastic processes from electrons on crystal nuclei, etc. Interesting possibilities for applications of the $\mathrm{RC}$ in the accelerator physics were discussed recently in Ref. [13].

The results also show that we have a new method for detecting the LP effect along axial directions in crystals where the multiple scattering is strongly enhanced $[11,14]$. In amorphous targets such an effect needs 10-100 TeV particles [15]. The LP effect is relevant for many physics applications like calorimeters for $\mathrm{TeV}$ machines (LHC, etc.). It suppresses pair production and thereby influences electromagnetic showers. This changes cosmic ray air showers [16] which are used to determine the total energy of ultrahigh-energy cosmic rays. The LP effect also has its analog in suppression of gluon emission from nuclear matter [17].

*On leave from Kurchatov Institute, 123182 Moscow, Russia.

[1] J. Lindhard, Mat. Fys. Medd. Dan. Vidensk. Selsk. 34, No. 14 (1965).

[2] A. H. Sørensen and E. Uggerhøj, Nucl. Sci. Appl. 3, 147 (1989).

[3] V. N. Baier, V. M. Katkov, and V. M. Strakhovenko, Nucl. Instrum. Methods Phys. Res., Sect. B 69, 258 (1992); Sov. Phys. Usp. 32, 972 (1989).

[4] X. Artru, Phys. Lett. A 128, 302 (1988).

[5] V. V. Tikhomirov, Nucl. Instrum. Methods Phys. Res., Sect. B 36, 282 (1989).

[6] V.G. Baryshevsky and I. Ya. Dubovskaya, Phys. Lett. 62A, 45 (1977).

[7] R. Medenwaldt et al., Phys. Rev. Lett. 63, 2827 (1989); Phys. Lett. B 242, 517 (1990).

[8] R. Medenwaldt et al., Phys. Lett. B 281, 153 (1992); Nucl. Instrum. Methods Phys. Res., Sect. B 103, 139 (1995).

[9] Yu. V. Kononets, J. Moscow Phys. Soc. 2, 71 (1992).

[10] L. D. Landau and I. Ya. Pomeranchuk, Dokl. Akad. Nauk SSSR 92, 535 (1953); 92, 735 (1953).

[11] Special issue on Channeling and Other Crystal Effects at Relativistic Energy, edited by H. H. Andersen, R. A. Carrigan, and E. Uggerhøj [Nucl. Instrum. Methods Phys. Res., Sect. B 119, 1-315 (1996)].

[12] In most papers the length $\bar{\ell}_{f}$ in the CFA was assumed to be the path over which the particle is deflected an angle $\gamma^{-1}$, i.e., $\bar{\ell}_{f} \approx a m c^{2} /\left|U_{0}\right|$, where $a$ and $U_{0}$ are the width and the height of the axial potential. This led to the inequality $\bar{\ell}_{f} \ll \ell_{c}(\hbar \bar{\omega})$ which is wrong at $\chi \lesssim 1$.

[13] Z. Huang, P. Chen, and R. D. Ruth, Phys. Rev. Lett. 74, 1759 (1995).

[14] J. F. Bak et al., Nucl. Phys. B302, 525 (1988).

[15] P. L. Anthony et al., Phys. Rev. Lett. 75, 1949 (1995).

[16] E. Konishi et al., J. Phys. G 17, 719 (1991).

[17] S. J. Brodsky and P. Hoyer, Phys. Lett. B 298, 165 (1993). 